\title{
Does Urethral Competence Affect Urodynamic Voiding Parameters in Women With Prolapse?
}

\author{
Ingrid Nygaard, ${ }^{1 *}$ Karl Kreder, ${ }^{2}$ Elizabeth Mueller, ${ }^{3}$ Linda Brubaker, ${ }^{3}$ Patricia Goode, ${ }^{4}$ \\ Anthony Visco, ${ }^{5}$ Anne M. Weber, ${ }^{6}$ Geoff Cundiff, ${ }^{7}$ and John Wei ${ }^{8}$ for the Pelvic Floor Disorders Network \\ ${ }^{1}$ Department of Obstetrics and Gynecology, University of Utah, Salt Lake City, Utah \\ ${ }^{2}$ Department of Urology, University of Iowa Carver College of Medicine, Iowa City, Iowa \\ ${ }^{3}$ Departments of Urology and Obstetrics and Gynecology, Loyola University Medical Center, Maywood, Illinois \\ ${ }^{4}$ Birmingham/Atlanta Geriatric Research, Education and Clinical Center, Birmingham Veterans Affairs Medical Center \\ and Department of Medicine, University of Alabama at Birmingham, Birmingham, Alabama \\ ${ }^{5}$ Department of Obstetrics and Gynecology, University of North Carolina at Chapel Hill, Chapel Hill, North Carolina \\ ${ }^{6}$ National Institute for Child Health and Human Development \\ ${ }^{7}$ Department of Obstetrics and Gynecology, University of British Columbia, Vancouver, Canada \\ ${ }^{8}$ Department of Urology, University of Michigan, Ann Arbor, Michigan
}

\begin{abstract}
Aims: To (1) compare voiding parameters and (2) correlate symptoms and urodynamic findings in women with pelvic organ prolapse (POP) and varying degrees of urethral competence. Methods: We compared three groups of women with stages II-IV POP. Groups 1 and 2 were symptomatically stress continent women participating in the Colpopexy and Urinary Reduction Efforts (CARE) trial; during prolapse reduction before sacrocolpopexy, Group $1(n=67)$ did not have and Group $2(n=84)$ had urodynamic stress incontinence (USI) during prolapse reduction. Group 3 participants $(n=74)$, recruited specifically for this study, had stress urinary incontinence (SUI) symptoms and planned sacrocolpopexy. Participants completed standardized uroflowmetry, pressure voiding studies, and validated symptom questionnaires. Results: Subjects' median age was 61 years, median parity 3 and $87 \%$ had stage III or IV POP. Fourteen percent of women in Group 3 demonstrated USI without, and 70\% with, prolapse reduction. Women in Groups 2 and 3 had more detrusor overactivity (DO) than Group 1 (17 and 24\% vs. 6\%, $P=0.02$ ) and detrusor overactivity incontinence (DOI) ( 15 and $8 \%$ vs. $0 \%, P=0.004$ ). Based on the Blaivis-Groutz nomogram, $60 \%$ of all women were obstructed. Post-void residual volume (PVR), peak flow rate, detrusor pressure at peak flow, voiding mechanisms, voiding patterns, obstruction and urinary retention did not differ among groups. Women in Group 3 had higher irritative and obstructive symptom scores than Group 1 or 2; neither score differed by presence of DO nor obstruction, respectively. Conclusion: Women with POP have significant rates of urodynamic obstruction and retention, independent of their continence status. Symptoms of obstruction and retention correlate poorly with urodynamic findings. Neurourol. Urodynam. 26:1030-1035, 2007. (c) 2007 Wiley-Liss, Inc.
\end{abstract}

Key words: obstruction; pelvic organ prolapse; urodynamics; voiding

\section{INTRODUCTION}

Pelvic organ prolapse (POP) is a complex condition often associated with both urinary incontinence and urinary retention. The studies that address urodynamic evaluation in women with POP have focused on uncovering urodynamic stress incontinence during prolapse reduction ${ }^{1-5}$ or defining obstructive voiding on pressure flow studies. ${ }^{6,7}$ Interpretation of these studies has been limited by their small sample sizes.

To better understand bladder function in women with prolapse, we conducted a prospective supplementary study to the NIH/NICHD's Pelvic Floor Disorders Network (PFDN) Colpopexy and Urinary Reduction Efforts (CARE) study. ${ }^{8}$ The CARE study enrolled women without symptoms of stress incontinence undergoing sacrocolpopexy for pelvic organ prolapse with the primary aim of determining if adding a Burch urethropexy at the time of surgery would reduce symptoms of stress incontinence 3 months post-operatively. Since stress urinary incontinence (SUI) symptoms occur in 25$100 \%$ of women with advanced prolapse, ${ }^{1,2,4-7}$ we recognized the opportunity to compare women enrolled in CARE with women who were also undergoing sacrocolpopexy for prolapse but who additionally had stress incontinence symptoms.

By studying a large group of women with POP who are similar except for their symptoms of stress incontinence, our overall aim was to better understand how concomitant urethral incompetence impacts patient symptoms and voiding parameters. Our hypothesis was that urethral incompetence as indicated by stress incontinence symptoms may modify the functional obstruction of pelvic organ prolapse.

The specific aims of this study were to compare voiding parameters and correlate symptoms and urodynamic findings in three groups of women with symptomatic pelvic organ prolapse (POP) who were planning sacrocolpopexy and who had varying degrees of urethral competence. We defined groups as follows: Group 1 (no SUI), women with no stress urinary

Heinz Koelbl led the review process

Abbreviations: POP, pelvic organ prolapse; SUI, stress urinary incontinence; USI, urodynamic stress incontinence; PVR, post-void residual volume; DO, detrusor overactivity; DOI, detrusor overactivity incontinence; UDI, urinary distress inventory; NIF, non-instrumented flow study; PFS, pressure flow study Grant sponsor: National Institute of Child Health and Human Development; Grant numbers: U01 HD41249, U10 HD41268, U10 HD41248, U10 HD41250, U10 HD41261, U10 HD41263, U10 HD41269, U10 HD41267.

${ }^{*}$ Correspondence to: Ingrid Nygaard, Department of Obstetrics and Gynecology, University of Utah College of Medicine, 30 North 1900 East, Room 2B 242, Salt Lake City, UT 84132. E-mail: Ingrid.nygaard@hsc.utah.edu Received 20 December 2006; Accepted 27 February 2007 Published online 16 July 2007 in Wiley InterScience (www.interscience.wiley.com)

DOI 10.1002/nau.20436 
incontinence (SUI) symptoms and no urodynamic stress incontinence (USI) during prolapse reduction; Group 2 (occult SUI), women with no SUI symptoms who demonstrated USI on reduction testing; Group 3 (overt SUI), women with SUI symptoms.

\section{METHODS}

Urodynamic data for this study were obtained from 298 women with stages II-IV pelvic organ prolapse. ${ }^{9}$ Of these, 225 were planning sacrocolpopexy and represent the three comparison groups; we enrolled an additional 73 women to provide descriptive data only (see below). Groups 1 and 2 consist of women with no or minimal subjective symptoms of stress urinary incontinence who participated in the Colpopexy and Reduction Efforts Trial $^{8}$ and underwent urodynamics prior to surgery. Group 3, recruited specifically for this study, consists of women with SUI symptoms who were planning to undergo a sacrocolpopexy. Women in Group 3 met all the inclusion/exclusion criteria for CARE except that they had symptomatic SUI, an exclusion criterion for CARE. As noted we also enrolled an additional 73 women (Group 4) for descriptive purposes only; these women had SUI symptoms but were not planning to undergo sacrocolpopexy. Because women with POP undergoing urodynamics who are not planning abdominal surgery differ demographically from our study groups, we decided a priori not to compare them to the other three groups, but to describe them in order to add to the scarce literature in this area.

We excluded women who were unable to provide informed consent or participate in a quality of life telephone interview in English, were currently pregnant or within 6 months postpartum, had neurological diagnoses that may affect voiding function such as multiple sclerosis or spinal cord injury.

Patients were considered stress continent (and thus eligible for CARE) if they responded "never" or "rarely" to six MESA ${ }^{10}$ stress incontinence questions: coughing hard, sneezing, lifting, bending, laughing, and walking briskly or jogging ${ }^{8}$ and stress incontinent if they responded "sometimes" or "usually" to any of these same MESA questions.

The make-up of the three comparative groups is summarized as follows:

(1) Group 1 (no SUI): 67 women randomly selected from women enrolled in the CARE study who had no subjective symptoms of SUI on the MESA and demonstrated no USI with prolapse reduction testing.

(2) Group 2 (occult SUI): 84 women randomly selected from women enrolled in the CARE study who had no subjective symptoms of SUI on the MESA, but who did demonstrate USI with reduction testing (i.e., urinary leakage from the urethral meatus with cough or valsalva).

(3) Group 3 (overt SUI): 74 women who were similar to CARE patients, i.e., they were planning sacrocolpopexy for repair of prolapse and they did have subjective symptoms of SUI on the MESA.

Women enrolled in this supplementary study completed questionnaires and procedures identical to those completed at baseline in CARE. Relevant to this study were: demographic and history questions, standardized pelvic organ prolapse quantification $^{11}$ examination by a certified research nurse, standardized urodynamic evaluation (described in detail below), and the pelvic floor distress inventory (PFDI). ${ }^{12}$ These survey instruments were completed via a telephone interview from the Quality of Life Interviewing Center at the University of Michigan.

Lower urinary tract storage symptoms (urgency, frequency, sensation) were obtained by positive responses and report of at least moderate bother to the items in the urinary distress inventory (UDI) of the PFDI ${ }^{12}$ (i.e., questions 17, 18, 19, 23, 26, $27,29,33,34,28)$. Voiding symptoms were obtained by positive responses and report of at least moderate bother to the items in the obstructive/discomfort scale of the UDI in PFDI $^{12}$ (i.e., questions $11,12,13,14,15,16$ ).

\section{Urodynamics Protocol}

Non-fluoroscopic urodynamic studies with external water pressure transducers were performed on all participants preoperatively. Uroflowmetry was done with participants in a seated position with a comfortably full bladder before urethral instrumentation. Post-void residual (PVR) urine volume was obtained by catheterization within $15 \mathrm{~min}$ of voiding. Urodynamics were rescheduled if dipstick urinalysis suggested infection. The uroflowmeter was calibrated in accordance with routine clinical practice standards and the scale was set to zero before each study. We did not repeat the uroflowmetry if the participant was unable to void or had a voided volume $<150 \mathrm{ml}$ as the applicability of this minimum voiding volume, in terms of study interpretation, is not known for women with pelvic organ prolapse.

The cystometrogram was performed with participants seated at a $45^{\circ}$ angle using the same multichannel urodynamic recorder as was used for the CARE protocol. External water transducer catheters $\leq 8$ French were used and zeroed to atmosphere at the superior level of the symphysis pubis prior to insertion. Intravesical pressure (Pves), intra-abdominal pressure (Pabd), and subtracted detrusor pressure (Pdet) were continuously recorded on a multichannel urodynamics recorder throughout the cystometrogram. The bladder was filled with saline or sterile water at $50 \mathrm{ml} / \mathrm{min}$. A rectal catheter was used to estimate intra-abdominal pressure. If the detrusor pressure was not between 0 and $5 \mathrm{~cm} \mathrm{H}_{2} \mathrm{O}$ during early filling, the ports were flushed and the equipment was rezeroed. Baseline intravesical pressure was recorded at the start of infusion. If a detrusor contraction occurred that caused a large volume of leakage during bladder filling at a volume less than $300 \mathrm{ml}$, the bladder was emptied and filling restarted at an infusion rate half of the original rate (i.e., $25 \mathrm{ml} / \mathrm{min}$ ).

At $300 \mathrm{ml}$ or maximum bladder capacity, whichever was lower, baseline intravesical pressure was recorded at rest. Participants then did a series of three coughs and three valsalva efforts, first with the prolapse out and then with each of two methods of prolapse reduction. Details of the prolapse reduction technique have been previously published..$^{13}$ Briefly, each site used two of five methods to reduce prolapse (hand, speculum, pessary, cotton swab, and ring forceps). Each site used the same two methods for the whole study. At $300 \mathrm{ml}$ bladder volume, the prolapse was reduced with the first and then the second of two methods assigned to each clinical site. At this point, the second prolapse reduction method was left in place and the bladder was filled to maximum capacity. The valsalva and cough sequences were repeated at maximum capacity with only the second prolapse reduction method.

If the participant had not leaked following valsalva and cough testing at maximum capacity, the transurethral catheter was removed and the participant was instructed to valsalva and cough with maximal effort. Any urine leakage was recorded. Regardless of whether leakage occurred, the prolapse reduction method was removed and the transure- 
thral catheter was replaced for the pressure flow voiding study.

The prolapse was not reduced at the time of the pressure flow study by the research staff and the patient was instructed to bear down or cough to encourage the prolapse to become maximal again prior to voiding. Urethral relaxation was not documented during the pressure flow voiding study.

In accordance with recommendation of the ICS Standardization Committee of Good Urodynamic Practice, ${ }^{14}$ the scaling of the tracings was standardized across centers as follows: One millimeter was equal to $1 \mathrm{sec}$ on the $x$-axis for both flow and volume; and on the y-axis, $1 \mathrm{~mm}$ was equal to $1 \mathrm{ml} / \mathrm{sec}$ for flow and equal to $10 \mathrm{ml}$ for volume. For the cystometrogram and pressure flow signals, a minimum scaling was set at $25 \mathrm{~cm} \mathrm{H} \mathrm{H}_{2} \mathrm{O} / \mathrm{cm}$ for pressure; $25 \mathrm{ml} / \mathrm{sec} / \mathrm{cm}$ for flow; and $1 \mathrm{~min} / 3 \mathrm{~cm}$ for the time axis. The signals of the non-instrumented uroflowmetry and pressure flow study were clearly labeled to simplify a central review. The tracings were annotated in accordance with the required data points.

Each urodynamic tracing including the non-instrumented uroflow (NIF) and pressure flow study (PFS) were independently reviewed centrally by three PFDN investigators (IN, KK, JW). The voiding pattern, derived independently from both the NIF and the PFS, was classified based on the tracing as continuous (flow rate reaches zero only at the end of void), interrupted (flow rate diminishes to zero at least once prior to end of void) or unable to determine. Voiding mechanism was classified based on the PFS as detrusor (any increase in Pdet during peak flow), strain (greater than or equal to $15 \mathrm{~cm} \mathrm{H}_{2} \mathrm{O}$ increase in Pabd during peak flow), detrusor and strain, neither, or unable to determine (catheter fell out or significant artifact). The individual interpretations of the three reviewers were collated and where all three differed for any given data element, the tracings were arbitrated with all three investigators present. During arbitration, the tracings were reviewed and majority vote was required to arrive at final determination for each data element.

\section{Description of Variables}

We assessed obstruction using the parameters of noninstrumented maximum flow rate $\left(\mathrm{Q}_{\max \mathrm{NIF}}\right)$ and maximum

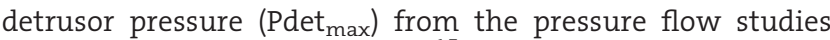
as outlined by Blaivis-Groutz. ${ }^{15}$ The void was defined as obstructed when values fell outside of the "unobstructed area" on the nomogram.

Urinary retention was defined as voiding $<75 \%$ of total bladder volume. Maximum bladder capacity was defined as the volume at which women stated they could no longer delay a trip to the bathroom.

\section{Statistical Analysis}

The three groups were compared by either a chi-square test when the measure was discrete or by an analysis of variance when the measure was continuous. Urodynamic data for the three groups were also compared after adjustment for age as a continuous measure and prior surgery for prolapse or incontinence. Correlations were computed to assess associations between measures.

With approximately 70 subjects per group there is almost $80 \%$ power to identify a difference of $0.5 \mathrm{SD}$ when testing a continuous outcome or a difference of $25 \%$ when testing a dichotomous outcome at a $5 \%$ level of significance.

\section{RESULTS}

Demographic characteristics and physical exam findings of the three groups, shown in Table I, were similar among cohorts except women in Group II were older $(P=0.022)$ and the proportion of women that had undergone previous incontinence surgery differed between all three groups $(P<0.001)$.

Only $13.7 \%$ of women with pre-operative symptoms demonstrated leakage during cough or valsalva testing without reduction; however, when prolapse reduction was performed in these same women, over two thirds (69.7\%) demonstrated leakage (Table II). Women without SUI symptoms and without USI on prolapse reduction (Group 1) were less likely to have detrusor overactivity (DO) or detrusor overactivity incontinence (DOI) on urodynamics than were women with occult or overt SUI $(6,16.7,23.6 \%$, respectively for DO; $P=0.016$ and $0,8.3,15.3 \%$, respectively for DOI; $P=0.004$ ).

The median PVR for the three groups combined was $46 \mathrm{ml}$ based on catheterization after the NIF and $25 \mathrm{ml}$ based on

TABLE I. Characteristics of Study Population

\begin{tabular}{|c|c|c|c|c|}
\hline Characteristic: mean \pm SD or $\mathbf{n}(\%)$ & No SUI $(N=67)$ & Occult SUI $(\mathbf{N}=\mathbf{8 4})$ & Overt SUI $(\mathbf{N}=74)$ & $P$-value \\
\hline Age & $58.2 \pm 10.4$ & $62.2 \pm 10.8$ & $57.2 \pm 14.4$ & $0.02^{\mathrm{a}}$ \\
\hline Parity & $\begin{array}{c}2.8 \pm 1.3, \text { median } 2.0 \\
\text { range } 1-8\end{array}$ & $\begin{array}{c}3.0 \pm 1.3, \text { median } 3.0 \\
\text { range } 1-7\end{array}$ & $\begin{array}{c}2.9 \pm 1.5, \text { median } 3.0, \\
\text { range } 0-8\end{array}$ & 0.75 \\
\hline Education & & & & 0.37 \\
\hline Less than high school & $6(9.0 \%)$ & $3(3.6 \%)$ & 7 (9.5\%) & \\
\hline Completed high school or equivalent & $26(38.8 \%)$ & $38(45.2 \%)$ & $22(29.7 \%)$ & \\
\hline Some college/Associate degree & $16(23.9 \%)$ & $26(31.0 \%)$ & $29(39.2 \%)$ & \\
\hline Completed 4 years of college & $9(13.4 \%)$ & $8(9.5 \%)$ & 7 (9.5\%) & \\
\hline Graduate/Professional degree & $10(14.9 \%)$ & $9(10.7 \%)$ & $9(12.2 \%)$ & \\
\hline POP-OQ stage & & & & 0.99 \\
\hline II & $8(11.9 \%)$ & $11(13.1 \%)$ & $10(13.5 \%)$ & \\
\hline III & $48(71.6 \%)$ & $61(72.6 \%)$ & $54(73.0 \%)$ & \\
\hline IV & $11(16.4 \%)$ & $12(14.3 \%)$ & $10(13.5 \%)$ & \\
\hline Prior surgery for POP & $18(26.9 \%)$ & $35(42.2 \%)$ & $30(41.1 \%)$ & 0.11 \\
\hline Prior surgery for UI & $0(\%)$ & $8(9.6 \%)$ & $19(25.7 \%)$ & $<0.001^{\mathrm{b}}$ \\
\hline Maximum anterior prolapse (cm) & $+3.0 \pm 2.5$ & $+3.2 \pm 2.6$ & $+2.8 \pm 2.8$ & 0.72 \\
\hline Maximum posterior prolapse $(\mathrm{cm})$ & $+2.6 \pm 3.0$ & $+2.4 \pm 3.4$ & $+2.0 \pm 3.4$ & 0.52 \\
\hline Maximum vaginal descent $(\mathrm{cm})$ & $+3.6 \pm 2.2$ & $+3.9 \pm 2.2$ & $+3.6 \pm 2.4$ & 0.75 \\
\hline
\end{tabular}

${ }^{a}$ Women with occult SUI are older than either of the other two groups.

${ }^{\mathrm{b}}$ All three groups differ. 
TABLE II. Urodynamic Findings

\begin{tabular}{|c|c|c|c|c|}
\hline Characteristic: mean \pm SD or $\mathbf{N}(\%)$ & No SUI $(\mathbf{N}=67)$ & Occult SUI $(\mathbf{N}=84)$ & Overt SUI $(\mathbf{N}=\mathbf{7 4})$ & $P$-value \\
\hline USI without prolapse reduction & $0(\%)$ & $9(11.0 \%)$ & $10(13.7 \%)$ & 0.01 \\
\hline USI with prolapse reduction ${ }^{\mathrm{a}}$ & $0(\%)$ & $84(100 \%)$ & $46(69.7 \%)$ & $<0.001$ \\
\hline Detrusor overactivity on cystometrogram & $4(6.0 \%)$ & $14(16.7 \%)$ & $17(23.6 \%)$ & 0.01 \\
\hline Detrusor overactivity incontinence on cystometrogram & $0(\%)$ & $7(8.3 \%)$ & $11(15.3 \%)$ & 0.02 \\
\hline \multicolumn{5}{|l|}{ Instrumented pressure flow study: voiding mechanism } \\
\hline Detrusor & $35(52.2 \%)$ & $47(56.0 \%)$ & $36(48.6 \%)$ & \\
\hline Strain & $1(1.5 \%)$ & $0(\%)$ & $3(4.1 \%)$ & \\
\hline Detrusor and strain & $23(34.3 \%)$ & $15(17.9 \%)$ & $13(17.6 \%)$ & \\
\hline Neither & $2(3.0 \%)$ & $5(6.0 \%)$ & $6(8.1 \%)$ & \\
\hline Unable to determine or unable to void & $6(9.0 \%)$ & $17(20.2 \%)$ & $16(21.6 \%)$ & \\
\hline \multicolumn{5}{|l|}{ Instrumented pressure flow study: voiding pattern } \\
\hline Continuous & $25(37.3 \%)$ & $33(39.3 \%)$ & $40(54.1 \%)$ & \\
\hline Interrupted & $38(56.7 \%)$ & $43(51.2 \%)$ & $29(39.2 \%)$ & \\
\hline Unable to determine or unable to void & $4(6.0 \%)$ & $8(9.5 \%)$ & $5(6.8 \%)$ & \\
\hline Obstruction by nomogram & $36(65 \%)$ & $42(60 \%)$ & $24(49 \%)$ & 0.23 \\
\hline Urinary retention on NIF (volume voided $<75 \%$ of bladder capacity) & $36(42.1 \%)$ & $30(38.0 \%)$ & $23(37.1 \%)$ & 0.84 \\
\hline Urinary retention on PFS (volume voided $<75 \%$ of bladder capacity) & $22(32.8 \%)$ & $21(27.3 \%)$ & $20(29.9 \%)$ & 0.77 \\
\hline $\begin{array}{l}\text { Post-void residual volume }\left(\mathrm{cm}^{3}\right) \text { (non-instrumented } \\
\quad \text { uroflowmetry) } \\
\text { (median) }\end{array}$ & $80.7 \pm 95.7(50.0)$ & $76.5 \pm 101.5(50.0)$ & $67.1 \pm 72.6(40.0)$ & 0.67 \\
\hline Post-void residual volume $\left(\mathrm{cm}^{3}\right)$ (pressure flow study) (median) & $90.5 \pm 118.2(33.0)$ & $74.6 \pm 129.5(25.0)$ & $74.1 \pm 96.7(30.0)$ & 0.64 \\
\hline Maximum flow (median) & $19.2 \pm 11.7(18.0)$ & $19.2 \pm 10.6(18.0)$ & $21.3 \pm 12.3(17.0)$ & 0.49 \\
\hline Detrusor pressure at maximum flow (median) & $31.6(24.8)(25.0)$ & $30.3(21.6)(26.0)$ & $26.8(22.1)(23.0)$ & 0.46 \\
\hline
\end{tabular}

${ }^{\text {a }}$ Significant due to definition of cohorts.

${ }^{\mathrm{b}}$ Derived from calculating maximum bladder capacity minus volume voided. When this difference was a negative number ( $\mathrm{N}=92$ ), the PVR was deemed $0 \mathrm{~cm}^{3}$.

calculation after the pressure flow study. Overall, 59.6\% (102/ 171) met the definition for obstruction. The median peak flow for all groups combined was $14 \mathrm{ml} / \mathrm{sec}$ during the NIF and $17 \mathrm{ml} / \mathrm{sec}$ during the PFS. The median detrusor pressure at peak flow was $25 \mathrm{~cm} \mathrm{H}_{2} \mathrm{O}$. The PVR, median peak flow rate, median detrusor pressure at peak flow, rates of urinary retention, and rates of obstruction were similar across the three groups (Table II).

Of the 186 women for whom a voiding mechanism could be determined, 118 (63.4\%) voided by detrusor contraction alone, $51(27.4 \%)$ voided with a combination of detrusor contraction and strain, 4 voided by strain alone, and 13 voided with neither strain nor detrusor contraction. We were unable to determine the voiding mechanism in 39 (17.3\%) women. There were no differences in voiding mechanism or voiding pattern by study group (Table II). There was no statistically significant difference in the voiding mechanisms whether or not women were obstructed, as defined by the nomogram.

We evaluated the association between urodynamic findings and bladder symptoms as reported on the PFDI. While women with overt SUI were more likely to have higher irritative and obstructive symptom scores, neither the irritative or obstructive symptom subscale score differed according to whether urodynamics revealed DO or obstruction, respectively (Table III). There was a weak correlation between the PVR from the pressure flow study and the UDI obstructive subscale score $(\mathrm{r}=0.25)$.

\section{DISCUSSION}

This is the largest study to rigorously compare the urodynamic findings of well-characterized women with advanced pelvic organ prolapse with and without stress incontinence symptoms. For decades, clinicians have assumed that advanced POP and obstruction commonly coexist. If obstruction is an entity of importance in women, a cohort with POP is the most logical clinical population to study.

The measure of obstruction in women (including women with POP) was initially modeled on male obstruction, although the entity of obstruction in women may be a different clinical phenomenon than obstruction in men. Our study highlights a high rate of obstruction, defined by one

TABLE III. Association Between Symptoms on the PFDI and Urodynamic Findings

\begin{tabular}{|c|c|c|c|c|c|}
\hline Characteristic: mean $\pm \mathbf{S D}$ (median) $\mathbf{N}$ & No SUI & Occult SUI & Overt SUI & $P$-value & Adjusted $P$-value ${ }^{\mathrm{a}}$ \\
\hline \multicolumn{6}{|l|}{ Irritative subscale score (median) ${ }^{\mathrm{b}}$} \\
\hline DO present & $25.6 \pm 24(18.8) 4$ & $26.1 \pm 16.9(32.5) 14$ & $46.1 \pm 22.2(47.5) 17$ & $0.02^{\mathrm{c}}$ & 0.05 \\
\hline DO absent & $17.0 \pm 15.4(15.0) 62$ & $21.4 \pm 16.9(18.8) 70$ & $39.9 \pm 24.3(37.5) 52$ & $<0.001^{\mathrm{d}}$ & $<0.001$ \\
\hline \multicolumn{6}{|l|}{ Obstructive subscale score (median) ${ }^{\mathrm{e}}$} \\
\hline Obstruction present & $32.0 \pm 18.4(27.9) 36$ & $44.5 \pm 22.8(45.2) 42$ & $45.2 \pm 28.5(42.3) 24$ & 0.03 & 0.05 \\
\hline Obstruction absent & $34.0 \pm 19.8(28.8) 18$ & $34.6 \pm 21.4(28.8) 28$ & $49.5 \pm 20.2(46.2) 23$ & 0.02 & 0.06 \\
\hline
\end{tabular}

${ }^{a}$ Adjusted for age (continuous) and prior surgery for prolapse or incontinence.

${ }^{\mathrm{b}}$ Irritative subscale scores did not differ according to whether or not DO was present.

${ }^{\mathrm{c}}$ Mean score for overt SUI group is higher than score for no SUI group.

${ }^{\mathrm{d}}$ Score for overt SUI group is higher than score for no SUI group and occult SUI group.

eObstructive subscale score did not differ according to whether or not obstruction (defined by the nomogram) was present. 
commonly used nomogram in women with POP, but this finding did not correlate with symptoms, regardless of degree of urethral competence.

The current ICS nomenclature does not provide terms for urodynamic findings during testing of women with POP. The storage phase term "urodynamic stress incontinence" does not discriminate as to whether advanced prolapse is reduced nor does it report preferred reduction techniques. Similarly, there are no recommendations for uninstrumented and instrumented voiding studies in women with prolapse. Aside from these nomenclature limitations, the urodynamic finding of "reduced" USI or "obstruction" are of uncertain clinical significance.

Most clinicians would assume that a woman with a weaker sphincter (as evidenced by her SUI symptoms) should have fewer obstructive symptoms. Indeed, we had a priori hypothesized that as the degree of urethral competence decreased from no SUI to occult SUI to overt SUI that obstruction, retention, irritative symptoms, and obstructive symptoms would decrease, because of the potential pressure release valve effect of the less competent urethra in the face of obstruction from prolapse. However, there is no evidence to support this widely held belief. Obstruction, as defined, is a voiding phase entity as opposed to stress incontinence which is a filling phase entity. Given that our findings did not, in fact, support our hypothesis, it is likely that urethral sphincter function during storage does not relate to sphincter function during emptying, when the normal sphincter relaxes. This would explain why maximum flow rates and detrusor pressures at maximum flow rates do not differ between women with and without stress incontinence regardless of their prolapse status (Table IV). ${ }^{15,16}$

That women with SUI have more irritative and obstructive symptoms than women without SUI may reflect a more endstage process in the evolution of pelvic floor disorders. Women with SUI were more likely to have had prior SUI surgery. We did adjust for this in our analyses, but the possibility remains that some dysfunction may have been caused by operative factors, such as denervation. We also did not measure urethral pressures in this study and so cannot comment on the correlation between actual pressures and symptoms.

Clinicians recommend treating elevated PVRs associated with advanced prolapse by reducing the prolapse, either with surgery or pessary, as this nearly universally returns the PVR to the normal range. ${ }^{17}$ Given this, it is reasonable to question what additional value can be gained from pressure flow voiding studies for women who already plan treatment of POP. Our evaluation examined whether voiding studies played a role in discriminating which women have storage phase abnormalities, such as stress incontinence to better understand bladder function in women with advanced prolapse. We did not find evidence to support nor recommend use a voiding phase study to "triage" the stress incontinence procedure. However, the value of a pressure flow study in patients with advanced prolapse undergoing a concurrent stress incontinence procedure remains unclear. There would be clinical value to such a voiding study if it were able to predict patients who develop retention postoperatively. Our study was not designed to answer this

\begin{tabular}{|c|c|}
\hline \multicolumn{2}{|l|}{ Characteristic: mean \pm SD or $\mathrm{N}(\%)$} \\
\hline Age (years) & $59.6 \pm 13.7$ \\
\hline Parity & $2.9 \pm 1.4$, median 3.0 , range $0-7$ \\
\hline \multicolumn{2}{|l|}{ POP-O stage } \\
\hline II & $32(43.8 \%)$ \\
\hline III & $36(49.3 \%)$ \\
\hline IV & $5(6.8 \%)$ \\
\hline Prior surgery for POP & $19(26.0 \%)$ \\
\hline Prior surgery for UI & $16(21.9 \%)$ \\
\hline USI without reduction & $11(15.1 \%)$ \\
\hline USI with prolapse reduced & $54(78.3 \%)$ \\
\hline USI only at capacity with prolapse reduced and catheter removed & $5(29.4 \%)$ \\
\hline $\mathrm{DO}$ & $11(15.3 \%)$ \\
\hline DO incontinence & $7(9.7 \%)$ \\
\hline Obstruction (by nomogram) & $33(57.9 \%)$ \\
\hline Retention (on non-instrumented uroflowmetry) & $26(40.6 \%)$ \\
\hline Peak flow $\left(\mathrm{cm}^{3} / \mathrm{sec}\right)$ & $22.5 \pm 11.5$ \\
\hline Detrusor pressure at peak flow $\left(\mathrm{cm} \mathrm{H}_{2} \mathrm{O}\right)$ & $34.6 \pm 22.1$ \\
\hline \multicolumn{2}{|l|}{ Voiding mechanism } \\
\hline Detrusor & $35(47.9 \%)$ \\
\hline Strain only & 0 \\
\hline Detrusor and strain & $16(21.9 \%)$ \\
\hline Neither & $6(8.2 \%)$ \\
\hline Unable to determine & $16(21.9 \%)$ \\
\hline \multicolumn{2}{|l|}{ Voiding pattern (pressure flow study) } \\
\hline Continuous & $36(49.3 \%)$ \\
\hline Interrupted & $30(41.1 \%)$ \\
\hline Unable to determine & $7(9.6 \%)$ \\
\hline \multicolumn{2}{|l|}{ Irritative symptom subscale score } \\
\hline DO present & $36.7 \pm 21.6$ \\
\hline DO absent & $28.1 \pm 22.4$ \\
\hline \multicolumn{2}{|l|}{ Obstructive symptom subscale score } \\
\hline Obstruction present (by nomogram) & $41.0 \pm 23.7$ \\
\hline Obstruction absent & $40.7 \pm 24.0$ \\
\hline
\end{tabular}


question and the anti-incontinence procedure was limited to Burch colposuspension that is generally associated with a low rate of post-operative retention. ${ }^{18}$ Given the increase in the use of mid-urethral mesh slings and the potentially higher rate of post-operative urinary retention, future studies are warranted to examine the value of uninstrumented uroflowmetry studies and pressure flow studies in predicting post-operative urinary retention associated with slings in patients undergoing surgery for advanced prolapse.

Our study is limited by the lack of a gold standard for obstruction in women. It is possible that alternative measuring techniques may be more useful than the ones selected in this study. Our study population is limited to women with advanced prolapse planning sacrocolpopexy so we cannot comment on the findings of "obstruction" in women with better vaginal support or other cause of obstruction, including iatrogenic causes.

Strengths of our study include the large sample size, multisite recruitment, standardized urodynamics procedures with independent secondary review of voiding studies, and use of validated condition-specific instruments to assess symptoms.

If future research finds that voiding abnormalities, including obstruction, are of clinical importance in women with POP, the appropriate diagnostic tests require careful validation and testing for clinical relevance.

\section{AUTHOR DISCLOSURES}

Karl Kreder-Equity interests: Merck; consultant: Pfizer; speaker honorarium: Pfizer, Lilly, Astellas, Merck, Boehringer; research grant: Pfizer, Lilly, Astellas. Elizabeth Mueller-Trial participation: Pfizer, Allergan. Linda Brubaker-Trial participation: Pfizer, Allergan; research grant: Pfizer. Patricia Goode-Consultant: Johnson \& Johnson; trial participation: Pfizer.

\section{REFERENCES}

1. Richardson DA, Bent AE, Ostergard DR. The effect of uterovaginal prolapse on urethrovesical pressure dynamics. Am J Obstet Gynecol 1983;146:901-5.

2. Rosenzweig BA, Pushkin S, Blumenfeld D, et al. Prevalence of abnormal urodynamic test results in continent women with severe genitourinary prolapse. Obstet Gynecol 1992;79:539-42.
3. Wall LL, Hewitt JK. Urodynamic characteristics of women with complete posthysterectomy vaginal vault prolapse. Urology 1994;44:336-41. discussion 341-2

4. Veronikis DK, Nichols DH, Wakamatsu MM. The incidence of low-pressure urethra as a function of prolapse-reducing technique in patients with massive pelvic organ prolapse (maximum descent at all vaginal sites). Am J Obstet Gynecol 1997;177:1305-13. discussion 1313-4.

5. Gallentine ML, Cespedes RD. Occult stress urinary incontinence and the effect of vaginal vault prolapse on abdominal leak point pressures. Urology 2001;57:40-4.

6. Romanzi L. Management of the urethral outlet in patients with severe prolapse. Curr Opin Urol 2002;12:339-44.

7. Chaikin DC, Groutz A, Blaivas JG. Predicting the need for anti-incontinence surgery in continent women undergoing repair of severe urogenital prolapse. J Urol 2000;163:531-4.

8. Brubaker L, Cundiff GW, Fine P, et al. Abdominal sacrocolpopexy with Burch colposuspension to reduce urinary stress incontinence. N Engl J Med 2006; 354:1557-66

9. Bump RC, Mattiasson A, Bo K, et al. The standardization of terminology of female pelvic organ prolapse and pelvic floor dysfunction. Am J Obstet Gynecol 1996;175:10-7.

10. Diokno AC, Brock BM, Brown MB, et al. Prevalence of urinary incontinence and other urological symptoms in the noninstitutionalized elderly. J Urol 1986;136:1022-5

11. Bump RC, Hurt WG, Theofrastous JP, et al. Randomized prospective comparison of needle colposuspension versus endopelvic fascia plication for potential stress incontinence prophylaxis in women undergoing vaginal reconstruction for stage III or IV pelvic organ prolapse. The Continence Program for Women Research Group. Am J Obstet Gynecol 1996;175:326-33. discussion 333-5.

12. Barber MD, Kuchibhatla MN, Pieper CF, et al. Psychometric evaluation of 2 comprehensive condition-specific quality of life instruments for women with pelvic floor disorders. Am J Obstet Gynecol 2001;185:1388-95.

13. Brubaker L, Cundiff G, Fine $P$, et al. A randomized trial of colpopexy and urinary reduction efforts (CARE): Design and methods. Control Clin Trials 2003;24:629-42.

14. Schafer W, Abrams P, Liao L, et al. Good urodynamic practices: Uroflowmetry, filling cystometry, and pressure-flow studies. Neurourol Urodyn 2002;21: 261-74.

15. Blaivas JG, Groutz A. Bladder outlet obstruction nomogram for women with lower urinary tract symptomatology. Neurourol Urodyn 2000;19: 553-64.

16. Long CY, Hsu SC, Wu TP, et al. Urodynamic comparison of continent and incontinent women with severe uterovaginal prolapse. J Reprod Med 2004, 49:33-7.

17. Fitzgerald MP, Kulkarni N, Fenner D. Postoperative resolution of urinary retention in patients with advanced pelvic organ prolapse. Am J Obstet Gynecol 2000;183:1361-3. discussion 1363-4.

18. Lefranc JP, Atallah D, Camatte S, et al. Longterm followup of posthysterectomy vaginal vault prolapse abdominal repair: A report of 85 cases. J Am Coll Surg 2002;195:352-8. 